\title{
Interactive web mapping of 90 Years of Fire History Across South Australia
}

\author{
Joshua Andrew Gore ${ }^{a}$, Stefan Peters ${ }^{a}$ * \\ ${ }^{a}$ School of Natural and Built Environments, University of South Australia, GPO Box 2471, Adelaide, South Australia 5001, \\ Australia; gorja007@mymail.unisa.edu.au; stefan.peters@unisa.edu.au \\ * Corresponding author
}

Keywords: Open Source, Interactive web mapping, Internet Cartography, Leaflet

Abstract:

Fire is a significant part of South Australian history and integral to the state's ecosystems. Small prescribed fires are an essential part of ecosystem management and health. However, large, uncontrollable bushfires during South Australia's hot, dry summers often cause loss of property, damage to the environment and fatalities. An awareness of this fire history enables effective management and encourages residents to better prepare for catastrophic fire events. The South Australian Country Fire Service provides data on the burn extents of more than 5500 prescribed and accidental fire events across the state from 1931 to 2018 . This work presents the development of an open-source interactive web mapping tool, allowing residents, managers, and other map users to intuitively explore the state's fire history without the need for advanced software and skills. This web map application aims to clearly show the state's complex fire history at any zoom level from the entire state to a medium sized rural property.

To clearly symbolise areas with extensive fire history, events are shown as both polygons and centroid points. Points are symbolised using scale dependant clustering with cluster symbols including counts. This communicates both the number of fires and the extent of area burnt for regions of interest at all zoom levels. Statistics for the state's fire districts are also portrayed using a choropleth symbology. Popups provide further information on all point and polygon data.
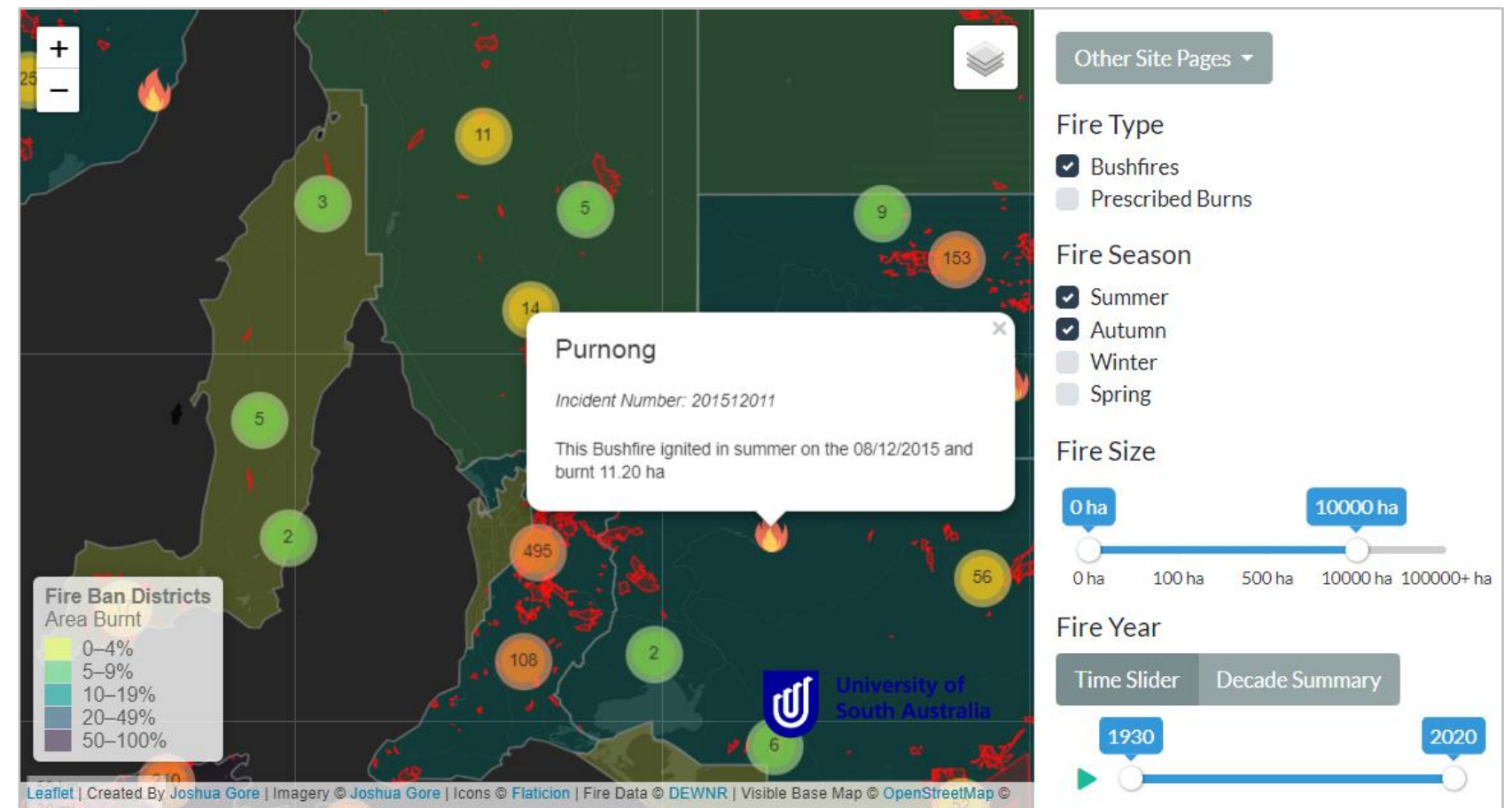

Figure 1. Screenshot of the Fire web map application - published at: https://bit.ly/2rAlnuV

All processing is done by the browser, with GeoJSON polygon geometry simplified and tiled using the geojson-vt library to ensure a responsive experience. Whilst resulting in a large initial download and requiring a mid-range PC for display this approach means data can be freely provided on static hosting with very little pre-upload processing required. Fire data can thus easily be updated as new incidents occur.

To allow responsive filtering of the over 5500 fire features an index system is implemented, using indices calculated both when data is loaded and on the fly in response to interface changes. The unique IDs given features within the fire GeoJSON are assigned to their leaflet layer representation. Lists of IDs of all features returning true when toggles are set to true are created on data load using a combination of the filter and map functions. Similar lists are created on the fly based on the status of range inputs. Intersect, union and difference functions are implemented using the Set data type 
allowing fast comparisons between filter results, visible, and not visible features to determine specific features to be shown or hidden.

This index system is utilised to provide a time series animation and a range of dual handle slider and checkbox interface elements allowing comprehensive data exploration based on event type, size, season, and year. When data is filtered by individual decade a textual historic summary of the decade is also displayed. The visualisation performs well on mid to high end desktop computers and thus demonstrates the potential client-side web technology has for providing comprehensive and accessible data exploration. 